\title{
Perfectly Monodisperse Microbubbling by Capillary Flow Focusing
}

\author{
Alfonso M. Gañán-Calvo and José M. Gordillo \\ Escuela Superior de Ingenieros, Universidad de Sevilla, Camino de los Descubrimientos s/n, 41092 Sevilla, Spain \\ (Received 12 April 2001; revised manuscript received 1 November 2001; published 11 December 2001)
}

Here we report a simple microfluidics phenomenon which allows the efficient mass production of micron size gas bubbles with a perfectly monodisperse and controllable diameter. It resorts on a selfexcited breakup phenomenon (which locks at a certain frequency) of a short gas microligament coflowing in a focused liquid stream. In this work, we describe the physics of the phenomenon and obtain closed expressions for the bubble diameter as a function of the liquid and gas properties, geometry, and flow parameters, from a large set of experimental results.

DOI: $10.1103 /$ PhysRevLett.87.274501

Microscopic gas bubbles appear and have countless applications in science and technology. In particular, fundamental medical applications of micron size bubbles range from ultrasound contrast agents to thrombus destruction, targeted drug delivery, tumor destruction, and even as an enhanced gene vector [1]. In addition, intravenous injection of a stabilized solution of sufficiently small bubbles might be used in acute lung disfunction. The size control of the microbubbles produced is critical in all these applications. Besides, exciting emergent technologies in Material Sciences, Chemical Engineering, Microelectronics, Biochemistry, Molecular Biology, etc., such as mesoscale self-assembly of newly developed smart materials, mesocrystals, microfabrication of tridimensional structures (e.g., [2-6]), molecular self-assembly (thousands of papers on it), DNA-driven assembly [7], etc., require the use of microtemplates, catalyzers, and microsubstrates as perfect and controllable as possible (and ideally equal in size and shape, like the atoms or molecules - see Figs. 1, 2, and 4) to perform such processes. Most of these processes are driven by surface tension or physicochemical interface forces and require a mass production of these microsubstrates to obtain a sufficient yield. On the other hand, chemical engineering processes as diverse as liquid aeration (e.g., water oxygenation, water purification [8,9]), liquid sterilization with $\mathrm{CO}_{2}$ or ozone, gas purification, or low temperature combustion of aqueous solutions of organic material (sewage) require the use of high performance gas-liquid diffusers. Food industries seek methods of controlling the density and texture of several substances, which could be easily accomplished if a gas could be microdispersed in these substances in the form of noncoalescing microbubbles.

In this work we propose a method to address these needs and provide the tools to use it.

1. Microfluidics phenomenon. - The aim of this paper is to report this phenomenon as a new microfluidic tool to mass generate highly controlled monosized microbubbles, and the experimental results so far obtained. In addition, we also make use of very recent theoretical results to obtain scaling laws and closed expressions for the bubble diameter.
PACS numbers: 47.55.Dz, 47.15.Hg, 47.60.+i, 47.27.Wg

Here we describe a capillary flow phenomenon which resorts on the focusing effect of a liquid stream trough a small orifice which provokes the tip streaming of a gas bubble attached to a feeding capillary tube. In our particular configuration a gas is continuously supplied from a capillary tube positioned upstream in the vicinity of an orifice through which a liquid stream is forced. At the mouth of the capillary tube, a cusplike attached bubble forms, from whose apex a steady gas ligament issues and is "focused" through the orifice by the surrounding liquid stream (see Fig. 1a). The gas ligament or hollow microjet then breaks up very soon into homogeneous size microbubbles (see Fig. 1b).

This configuration is analogous to the one described in [10] where a similar geometry is used as a liquid ligament generator. In the present work, however, a gas ligament

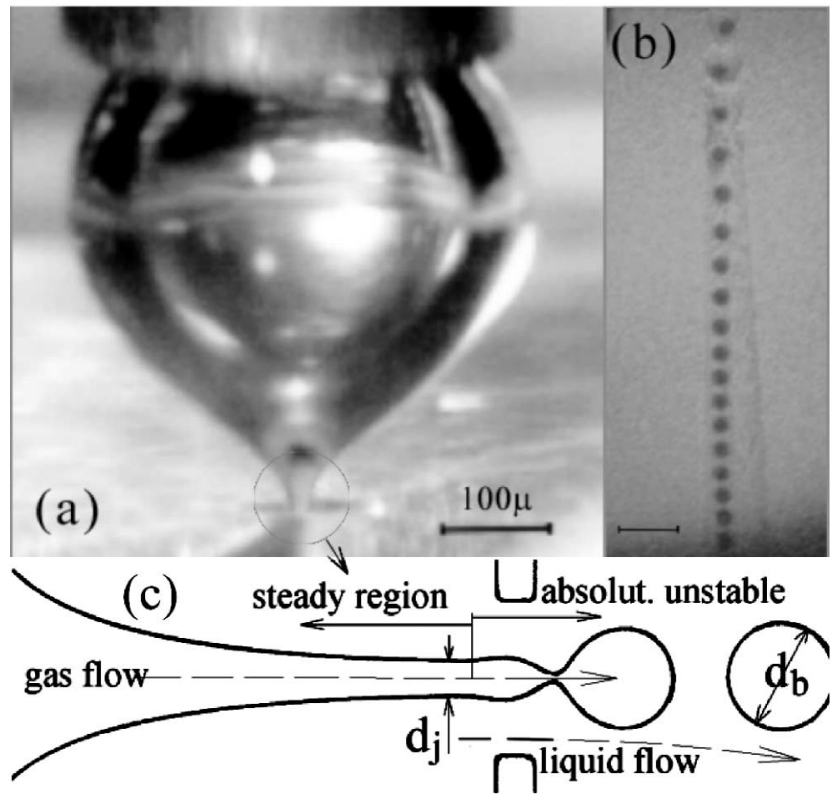

FIG. 1. (a) Cusplike bubble, attached to a capillary gas-feeding tube, from whose cusp a gas ligament issues through the orifice placed in front of the capillary. (b) Stream of gas bubbles issuing from the orifice. Picture taken with an exposure time of $1 \mu \mathrm{s}$. (c) Sketch of the region about the exit orifice, showing the steady and absolutely unstable regions of the gas ligament. 
surrounded by liquid is produced. Thus, the gas stream is surrounded by a much slower and denser coflowing liquid stream, and the physics of the gas ligament breakup phenomenon is dramatically different from that previously described process (here, equal size microbubbles are produced at a stunningly constant frequency): in particular, the gas ligament is so short that it is not observed at the exit of the orifice; on the contrary, a row of exactly equal, equidistant microbubbles is expelled from it (Figs. 1b, 2a, 2b). The physical explanation of the radically different behavior of a laminar gas ligament from a laminar liquid ligament results on the absolutely unstable nature of the gas ligament [11], contrarily to the convectively unstable behavior of liquid ligaments. Thus, the absolute instability of the gas ligament provokes its rapid breakup into microbubbles, but not only this: the nonlinear evolution of the local breakup of the ligament at the orifice involves a "self-excited" globally stable nonlinear saturation state (a limit cycle) with a saturated limit cycle amplitude [12]. This nonlinear phenomenon involves a strong self-locking of the breakup frequency, which yields the observed stunning regularity of the microbubbles produced (see Figs. 2 and 4). In one of the configurations shown in Fig. 2, where hollow monodisperse droplets can be produced (details in Fig. 3), one of the interesting features is the control on the particle density, and thus on the particle's aerodynamic diameter. In particular, for large enough gas to liquid flow rates (Fig. 3, left), one can obtain very light hollow microdroplets - such as "soap microbubbles" - which provide a small aerodynamic diameter while conveying a large surface area, of interest for many applications in drug delivery, chemistry, combustion, etc.

In other experiments, we have managed to form perfectly ordered lattices of bubbles using their perfect

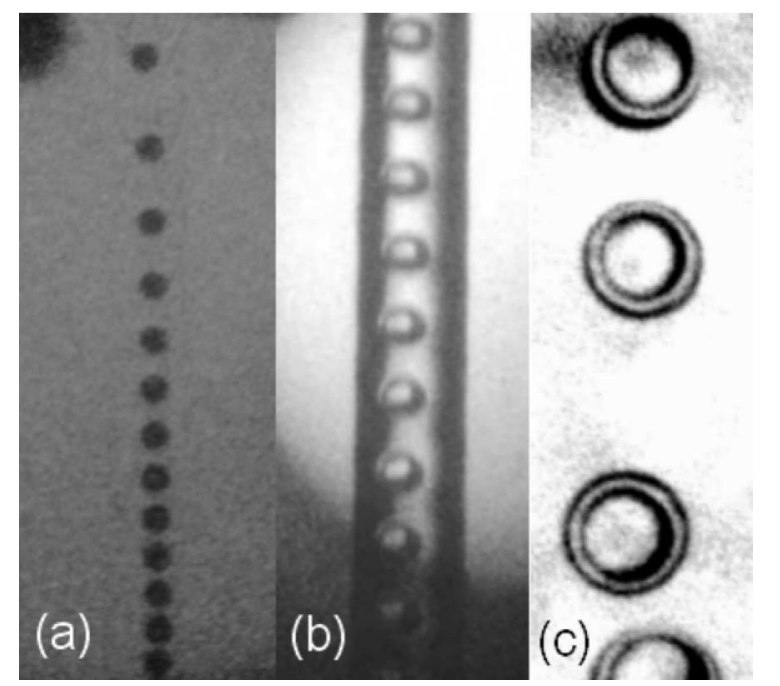

FIG. 2. (a) The liquid used to focus the gas is expelled into the same liquid. (b) The liquid with the gas bubbles is expelled into air, and a gas filled liquid jet with the diameter of the orifice is produced. This liquid jet eventually breaks up into equal-size gas filled microcapsules (c). Pictures taken with an exposure time of $11 \mu \mathrm{s}$. Arbitrary parameters (liquid: water $+20 \%$ ethanol). monodispersity (size deviations under steady conditions simply resulted zero with our measuring instruments, see Fig. 4). Because of the size controllability and potential mass productivity of this simple method, it might be of an extraordinary interest in the design of, for example, optical filters or highly structured, light bulk materials that can be formed from one, two, or a specific multiplicity of bubble sizes to achieve a certain ordered bulk structure for lightness and specific, isotropic or anisotropic mechanical resistance.

2. Experimental analysis. - Although we have observed stable microbubbling using orifice diameters $D$ from 500 down to $30 \mu \mathrm{m}$ and liquid viscosities $\mu_{l}$ from $10^{-3}$ to $10^{-1} \mathrm{~Pa} \cdot \mathrm{s}$, here we report a total of only 280 carefully measured bubble diameters $d_{b}$ for $D=100$ and $210 \mu \mathrm{m}$, liquid flow rates $Q_{l}$ from about 24 to $310 \mu \mathrm{l} / \mathrm{s}$, and gas (air) flow rates $Q_{g}$ from about 0.2 to $40 \mu \mathrm{l} / \mathrm{s}$ measured at atmospheric pressure and temperature of $298 \mathrm{~K}( \pm 0.5 \mathrm{~K})$. At this pressure and temperature, air density is $\rho_{g}=1.2 \mathrm{~kg} / \mathrm{m}^{3}$ and its viscosity is $\mu_{g}=1.8 \times 10^{-5} \mathrm{~Pa} \cdot \mathrm{s}$. The gas flow rates have been introduced by a high precision Harvard Apparatus syringe pump with Hamilton Gastight syringes of 0.1, 0.5, and $3 \mathrm{ml}$. In some cases, the liquid is pumped from a pressurized container, whose pressure is controlled by a Rexroth precision electronic valve. The liquid flow rate is then measured with a digital weight and a clock. In other cases (small $D$ ) the liquid is injected with another syringe pump.

Seven different liquids (water-ethanol and waterglycerol mixtures) with viscosities $\mu_{l}$ ranging from 1.2 to $30 \mathrm{mPa} . \mathrm{s}$, surface tensions from 35 to $57 \mathrm{mN} / \mathrm{m}$, and liquid densities from 795 to $1200 \mathrm{~kg} / \mathrm{m}^{3}$ have been used in this study. Monodisperse microbubbles with diameters $d_{b}$ ranging from about 5 to $120 \mu \mathrm{m}$ have been measured using a long working distance Nikon zoom microscope (maximum magnification of $360 \times$ measured at a $14 \mathrm{in}$. Sony monitor) and either a high speed camera 4Quick from Stanford Computer Optics, or a Sony IRIS CCD camera. In the first case, the bubble motion can be frozen and its diameter determined. In the last case, the displacement of the perfect microbubble row produces a sufficiently high contrast "cylindrical" image to take accurate diameter $d_{b}$ measurements on the monitor.

In order to gain knowledge about the fluidic regimes present in this phenomenon, let us define the relevant nondimensional fluidic parameter: a comparison of inertia

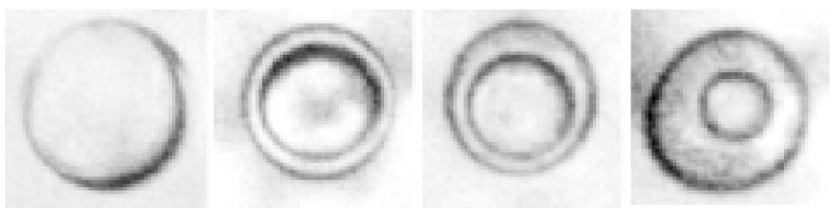

FIG. 3. Four examples of gas filled microcapsules that can be produced by this technique, by controlling the injected gas-liquid flow rates ratio $Q_{g} / Q_{l}=8,0.5,0.1,0.03$ (from left to right). Liquid: water $+20 \%$ ethanol. 


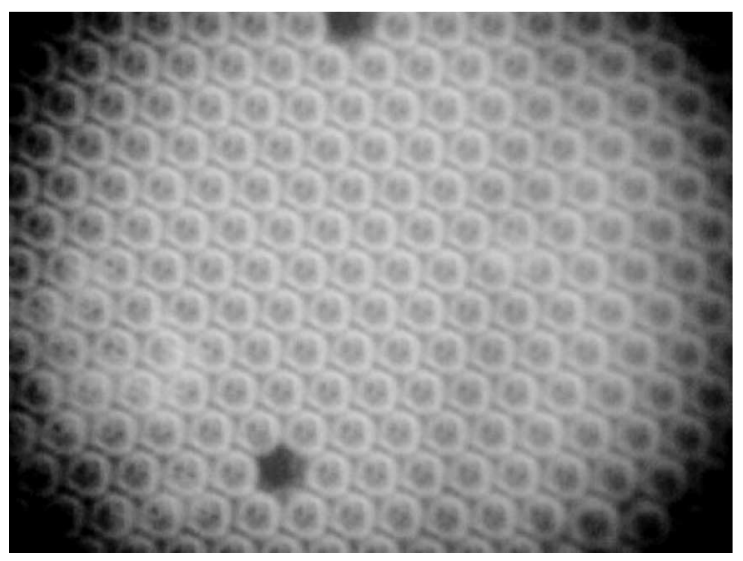

FIG. 4. When our microbubbles rise and settle, they form in many cases a "mesocrystal foam" or lattice. Bubbles are virtually equal in size, here $75 \mu \mathrm{m}$. Arbitrary parameters (liquid: water $+45 \%$ glycerol).

to viscous forces for both liquid and gas streams, given by the Reynolds number, can be written as

$$
\operatorname{Re}_{i}=\rho_{i} Q_{i} /\left(\mu_{i} D\right),
$$

where subindex $i$ stands for $l$ or $g$.

First, $\mathrm{Re}_{l}$ range from about 40 to about 1000 in all our experiments. These values are consistent with the physical fact that the liquid flow forms a well-defined jet at the exit (see Figs. 1b and 2), which should exert a sufficient momentum to convey and disperse the bubbles (otherwise they coalesce or do not form at all). Thus, the core of the liquid stream before and across the orifice (before the bubble formation) can be reasonably considered potentially highly convergent and laminar. On the other hand, though, $\mathrm{Re}_{g}$ range from about 0.07 to 14 , which means that we can have a gas flow ranging from a nearly creeping regime to a nearly potential one with a thick gas boundary layer at the gas-liquid interface.

3. Theoretical analysis. - As in the study of Oguz and Prosperetti [13] on the analysis of the regular bubbling from a submerged nozzle, the success in the physical description of the present phenomenon necessarily involves the identification of the relevant time governing the process. Here, however, because of the small sizes involved, gravity is negligible. Besides, the gas ligament rising the bubbles is not directly attached to a capillary tube as in [13], but there is a genuine focusing region (see Fig. 1) whose length is given by the exit orifice diameter $D$ at which the meniscus size varies from hundreds of microns (the capillary tube diameter $D_{1}$ ) down to just a few microns (the ligament diameter just before breakup at the orifice exit $d_{j}$ ). The phenomenon loses memory of the feeding tube size at the focusing orifice region. Then, there must be a well-defined cause of the time regularity observed, independent of the feeding tube and mass forces. In fact, the linear stability analysis [11] of the gas ligament suggests that the absolute instability growth rate of perturbations at the gas ligament, given by $t_{c}=d_{j} D^{2} / Q_{l}$, provides the relevant time of the process [14]. Then, the observed ligament breakup frequency $\omega$ should be $\omega \sim 1 / t_{c}$. When $\operatorname{Re}_{g}$ is large, the nondimensional breakup frequency $\Omega=$ $\omega t_{c}$ depends on the boundary layer thickness $\delta$ [11], and then $\Omega=\Omega\left(\operatorname{Re}_{g}\right)$. On the other hand, for small $\operatorname{Re}_{g}$, since the gas velocity profile should become independent of $\mathrm{Re}_{g}$, $\Omega$ should become independent of $\operatorname{Re}_{g}$ too. Consequently, the bubble diameter can be expressed as

$$
d_{b}=\left[6 Q_{g} /(\pi \omega)\right]^{1 / 3}=\left(\frac{Q_{g}}{Q_{l}}\right)^{1 / 3} D^{2 / 3} d_{j}^{1 / 3} f\left(\operatorname{Re}_{g}\right),
$$

where $f=[6 /(\pi \Omega)]^{1 / 3}$. In the following we outline the way to solve $d_{j}$ theoretically in order to find a predicting law for $d_{b}$. Assuming that $d_{j} \ll D$ (which holds in all our experimental conditions) and from Bernoulli's law (consistently with the Reynolds numbers involved), the pressure drop in the liquid from the region upstream of the orifice to the exit region is given by $\Delta P_{l}=1 /$ $2 \rho_{l}\left[4 Q_{l} /\left(\pi D^{2}\right)\right]^{2} \phi_{l}\left(\operatorname{Re}_{l}, L / D\right)$, where $L$ is the thickness of the plate with the orifice, and $\phi_{l}=1+29.6 /$ $\operatorname{Re}_{l}(1+1.7 L / D)$ [15]. On the other hand, if one takes into account the effect of the surface tension, the gas pressure drop at the ligament with diameter $d_{j}$ is given by $\Delta P_{g}=\Delta P_{l}-2 \gamma / d_{j}$. Neglecting compressibility effects (applicable in our experimental pressure ranges), its expression in terms of the gas momentum is $\Delta P_{g}=1 / 2 \rho_{g}\left[4 Q_{g} /\left(\pi d_{j}^{2}\right)\right]^{2} \phi_{g}\left(\operatorname{Re}_{g}\right)$, where function $\phi_{g}$ accounts for the viscous effects in the gas flow. Owing to the particular gas ligament geometry and to the fact that $\operatorname{Re}_{g}$ are sensibly smaller than $\mathrm{Re}_{l}, \phi_{g}\left(\mathrm{Re}_{g}\right)$ is in general a function different from $\phi_{l}\left(\operatorname{Re}_{l}\right)$. Thus, one obtains an implicit expression for the ligament diameter at the orifice exit, just before breakup:

$$
d_{j}=\left(\frac{\rho_{g}}{\rho_{l}}\right)^{1 / 4}\left(\frac{Q_{g}}{Q_{l}}\right)^{1 / 2} D\left(1-\frac{\pi^{2} \gamma D^{4}}{4 d_{j} \rho_{l} Q_{l}^{2}}\right)^{-1 / 4}\left(\frac{\phi_{g}}{\phi_{l}}\right) .
$$

where $\phi_{g} \sim O(1)$ depends on $\operatorname{Re}_{g}$ only. Substituting (3) into (2), one observes that $d_{b}$ scales as $\left(\phi_{l} / \phi_{g}\right)^{1 / 12}$, and thus it should result in being rather insensitive to $\phi_{g}$. Then, we will simply use $\phi_{g}=1$ [16] without any further refinements in the analysis of our experiments, absorbing into $f\left(\operatorname{Re}_{g}\right)$ the small deviations of $\phi_{g}^{1 / 12}$ from unity.

4. Analysis of the experimental results and scaling law. - To validate our previous analysis, we measured $d_{b}$, $Q_{g}, Q_{l}$, and calculated $d_{j}$ using expression (3). In Fig. 5 we plot the resulting values of $f\left(\operatorname{Re}_{g}\right)$ given by expression (2). The plot provides $f \simeq 2.9$ for small $\operatorname{Re}_{g}$, which smoothly tends to $f=3.15 \mathrm{Re}_{g}^{-0.167 \pm 0.003}$ for $\mathrm{Re}_{g}>6$ (regression coefficient above 98\%), which justifies our primary assumptions about the leading times and nature of the process governing our phenomenon.

To conclude this story, although Eqs. (2) and (3) now provide closed expressions to accurately calculate $d_{b}$, one may immediately note that expression (2) is quite insensitive to fluid properties. This fact is consistent with the surprising agreement between our following proposed, easy to remember expression for $d_{b}$, 

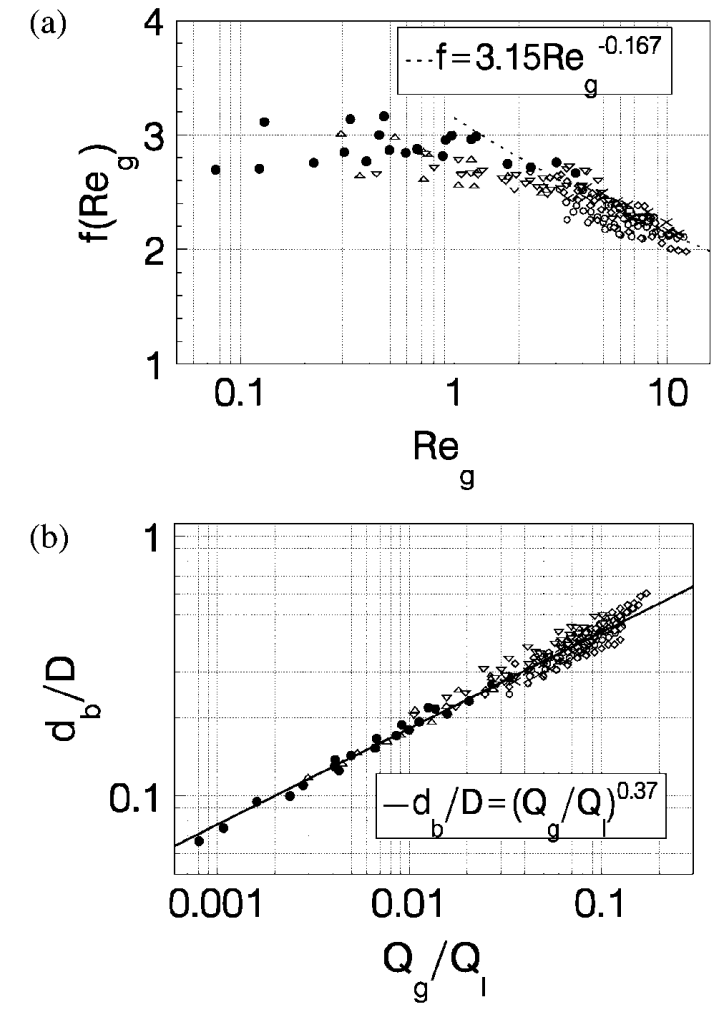

FIG. 5. (a) Experimental values of function $f\left(\operatorname{Re}_{g}\right)$. (b) Comparison of expression (4) with experimental values. Data points

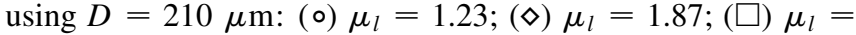
2.35; $(\triangle) \mu_{l}=10 ;(\bullet) \mu_{l}=30$. Data points using $D=$ $100 \mu \mathrm{m}:(\nabla) \mu_{l}=5$; $(\times) \mu_{l}=10$. Viscosity unit: cpoise $\left(10^{-3} \mathrm{~Pa} \cdot \mathrm{s}\right)$.

$$
d_{b} / D \simeq\left(Q_{g} / Q_{l}\right)^{0.37 \pm 0.005}
$$

and the experimental values, plotted in Fig. 5. This proposed expression can be very useful from the engineering point of view for its simplicity. Nota bene errors less than about $15 \%$ and the absence of fluid properties in this expression.

This work is supported by the Spanish Ministry of Science and Technology, and by Kraft Foods, Inc. Thanks must be given to J.L. Sampedro-Fernández, M. Hoc, N. Ouarty, T. Prevost, V. Marandat, and S. Vidal for their highly valuable assistance in experiments, and to M. Pérez-Saborid and J.M. López-Herrera for useful suggestions.

[1] Editorial, Physics News in 2000, APS News 10, No. 2, Suppl. 7 (2001).

[2] W. T. S. Huck, J. Tien, and G. M. Whitesides, J. Am. Chem. Soc., 120, 8267 (1998).

[3] A. Terfort, N. Bowden, and G. M. Whitesides, Nature (London) 386, 162 (1997).
[4] A. Terfort and G. M. Whitesides, Adv. Mater. 10, 470 (1998).

[5] M. Srinivasarao, D. Collings, A. Philips, and S. Patel, Science 292, 79 (2001).

[6] S. S. Jenekhe and X. L. Chen, Science 283, 372 (1999).

[7] E. Winfree, Nature (London) 394, 539 (1998).

[8] Y. Chung, Y. C. Choi, Y.H. Choi, and H.S. Kang, Water Res. 34, 817 (2000).

[9] M. Lundh, L. Jonsson, and J. Dahlquist, Water Res. 34, 21 (2000).

[10] A. M. Gañán-Calvo, Phys. Rev. Lett. 80, 285 (1998).

[11] J. M. Gordillo and A. M. Gañán-Calvo, and M. PérezSaborid, Phys. Fluids 133839 (2001).

[12] Analogous to the "hot jet dynamics," see P. A. Monkewitz and K. D. Sohn and AIAA J. 26, 911 (1988); P. Huerre and P. A. Monkewitz, Ann. Rev. Fluid Mech. 22, 473 (1990).

[13] H. N. Oguz and A. Prosperetti, J. Fluid Mech. 257, 111 (1993).

[14] Other times such as the ones associated to the viscous or nonviscous pinch-off of the initial ligament under surface tension only - see, for example, J. Eggers, Phys. Rev. Lett. 71, 3458 (1993); M. P. Brenner, J. R. Lister, and H. A. Stone, Phys. Fluids 8, 2827 (1996); I. Cohen, M. P. Brenner, J. Eggers, and S. R. Nagel, Phys. Rev. Lett. 83, 1147 (1999) — resulted in this particular phenomenon inconsistent with the measured $t_{c} \sim 1 / \omega$ values. We concluded that $t_{c}$ should be related to a rapid instability growth rate which pinches the ligament down to sizes where eventually viscous or inertia processes under surface tension finally become fast enough, but cannot be dominant.

[15] Z. Dagan, S. Weinbaum, and R. Pfeffer, J. Fluid Mech. 115, 505 (1982).

[16] A more or less straightforward rationale to obtain $\phi_{g}$ can be outlined. For large $\mathrm{Re}_{g}$, the gas flow is almost inviscid at the ligament, and then $\phi_{g}=1$ independently of its geometry. On the other hand, for small $\mathrm{Re}_{g}$ one has $\phi_{g}=k \mathrm{Re}_{g}^{-1}$. To solve the constant $k$, the viscous gas flow in the cusp-jet geometry should be solved. A first approximation to the gas cusp-jet geometry before the ligament breakup is the shape of liquid stream tube whose diameter is $d_{j}$ at the exit orifice and is given by the well-known solution of the inviscid liquid flow about the exit orifice in prolate spheroidal coordinates $(\xi, \eta, \phi)$ where $\eta=$ const are the stream tubes [see P. M. Morse and H. Feshbach, Methods of Theoretical Physics (Cambridge University Press, Cambridge, 1953)]. However, there is a serious restriction for the physical applicability of this rationale. In fact, the small diameter of the steady ligament (not to mention the pinch-off region), which is hardly larger than 10 times the molecular mean free path in the experimental cases where $\operatorname{Re}_{g}$ is small, and the large gas velocities in the gas ligament make it very questionable to consider a local thermodynamic quasiequilibrium state and the use of the no-slip boundary condition at the gas-liquid surface, and thus, even for small, $\mathrm{Re}_{g}$, the gas velocity profile in the ligament can very plausibly be assumed nearly flat, which readily yields $\phi_{g} \simeq 1$. 